\title{
A pilot study of solvent-based cleaning of yellow ochre oil paint: effect on mechanical properties
}

\author{
Ashley Amanda Freeman ${ }^{1 *} \mathbb{D}$, Judith Lee ${ }^{2}$, Cecil Krarup Andersen ${ }^{3}$, Naoki Fujisawa ${ }^{1}$, Michał Łukomski ${ }^{1}$ \\ and Bronwyn Ormsby ${ }^{2}$
}

\begin{abstract}
Nanoindentation and dynamic mechanical analysis were used to measure changes in the surface and bulk mechanical properties of Winsor \& Newton Yellow Ochre oil paint films following exposure to deionized water, aliphatic mineral spirits (Shellsol D40 ${ }^{\mathrm{TM}}$ ), and D5 silicone solvent (decamethylcyclopentasiloxane). Yellow ochre paint films were exposed to the selected solvents by 24-h immersion, and sponge-cleaning. 24-h immersion in deionised water and Shellsol D40 caused measurable changes to bulk and surface mechanical properties. However, there were no measurable changes to the bulk or surface mechanical properties following sponge cleaning.
\end{abstract}

Keywords: Modern oil paints, Cleaning, Winsor \& newton yellow ochre oil paint, Nanoindentation, Dynamic mechanical analysis

\section{Introduction}

Twentieth and twenty-first century oil paintings are associated with a range of challenging ageing and degradation phenomena [1-7]. For modern and contemporary oil paintings that are often left unvarnished and displayed unglazed, it can be challenging to remove surface-dirt that sits directly on the painted surface, whilst preserving as far as possible the appearance of the surface and physicochemical integrity of the artwork. This is particularly the case for paints with soft and/or deteriorated surfaces such as acrylic dispersion-based paints and some modern oil paints which can be vulnerable to the effects of solvents and mechanical action $[8,9]$.

The response of vulnerable painted artworks to surface-cleaning will naturally be influenced by paint formulation, the artists' materials and technique, age of the artwork, environmental exposure history, and past conservation treatment. The choice of solvents, method of

*Correspondence: ashley.freeman84@gmail.com

${ }^{1}$ The Getty Conservation Institute, Los Angeles, USA

Full list of author information is available at the end of the article solvent-delivery and associated mechanical action are important parameters [10-14]. Environmental conditions experienced during cleaning such as air flow, temperature, relative humidity and pressure may also affect both the behaviour of the solvents, and the response of the paint film. In order to help inform treatment decisions, it is important to understand the relative significance and likely effects of such factors on paint properties.

The extensive body of studies into the effects of solvents on oil paint has been the subject of a recent review [15]. Solvent-induced swelling and leaching of oil paint is a well-documented phenomenon [16, 17] and associated models have been proposed to help predict solvent-paint interactions [11, 12]. Stress-strain measurements have shown that solvent-induced leaching of the mobile constituents [18] of oil paint films leads to a general increase in bulk paint stiffness, particularly following prolonged immersion [10, 19]. Similar studies have been carried out on artists' dispersion paints [11, 12]. Studies that evaluate novel cleaning systems and methodologies designed to address specific challenges, often consider soil-removal efficacy, clearance, and surface integrity, supported by

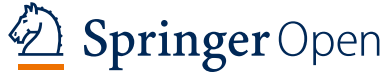

(c) The Author(s) 2021. This article is licensed under a Creative Commons Attribution 4.0 International License, which permits use, sharing adaptation, distribution and reproduction in any medium or format, as long as you give appropriate credit to the original author(s) and the source, provide a link to the Creative Commons licence, and indicate if changes were made. The images or other third party material in this article are included in the article's Creative Commons licence, unless indicated otherwise in a credit line to the material. If material is not included in the article's Creative Commons licence and your intended use is not permitted by statutory regulation or exceeds the permitted use, you will need to obtain permission directly from the copyright holder. To view a copy of this licence, visit http://creativeco mmons.org/licenses/by/4.0/. The Creative Commons Public Domain Dedication waiver (http://creativecommons.org/publicdomain/ zero/1.0/) applies to the data made available in this article, unless otherwise stated in a credit line to the data. 
high resolution imaging of the very paint surface that is most vulnerable during treatment $[6,14,20,21]$. However very few analytical techniques are available that have the sensitivity to study the effect of surface cleaning (involving the short exposure-times that is more typical of conservation practice), on the mechanical properties of the uppermost microns of paint surfaces. Nanoindentation paired with Dynamic Mechanical Analysis (DMA) and Dynamic Vapour Sorption (DVS) have recently proven useful when looking at the uppermost surface of a paint film and the local effect that solvent immersion and fluctuations in relative humidity have on oil paint [22].

This paper analyses changes in the mechanical properties of naturally aged yellow ochre oil paint films, following immersion and sponge-cleaning using selected solvents. Winsor \& Newton (W\&N) Yellow Ochre Artists' Oil Colour was selected for consistency with other related studies into modern oil paints, that have shown it to be associated with the development of water sensitivity [7], and vulnerable medium-rich surfaces that can complicate surface-cleaning [14]. Dynamic mechanical analysis and nanoindentation were used to investigate modifications to bulk and surface mechanical properties. SEM imaging and cross-sectional analysis provided complementary information about topographic alterations, and surface properties vital for interpretation of nanoindentation measurements.

The yellow ochre films were exposed to three solvents with varying properties: deionized water, the hydrocarbon solvent Shellsol D40, and the cyclic silicone solvent known as D5 (decamethylcyclopentasiloxane). These were selected as they form the basis of many solventcleaning strategies used in modern and contemporary art conservation that have been trialled on both acrylic dispersion and sensitive oil paints [14], as opposed to previous studies, which focused on more aggressive solvents $[13,19]$. Deionised water is widely used in conservation as one of the most inherently efficient options for soiling removal. However, when used unmodified (i.e. not $\mathrm{pH}$ and conductivity adjusted or buffered), is known to ehance the swelling of acrylic paints [23] with concomitant extraction/removal of surfactants [24]. Similarly, water is known to swell and extract mobile components from water sensitive modern oil paints [2] and to dissolve magnesium sulphate heptahydrate and other soluble salts where present [7]. Shellsol D40 is used as the non-polar (oil) component for the 'Series 3b' water-in-oil microemulsions that were developed through a Tate-Dow-Getty collaboration around the challenges associated with cleaning acrylic painted surfaces [25], which also show potential for the cleaning of water sensitive oil paints $[6,14,20]$. Silicone solvents such as D5, are also being increasingly used as cleaning systems for water-sensitive substrates both as a hydrophobic barrier [26] and/or as part of Pickering emulsifier formulations [27, 28].

In following sections, further details about selected materials (paint and solvents), applied treatments (immersions and sponge cleaning) as well as scientific methods are provided.

\section{Experimental \\ Paint samples}

Winsor \& Newton (W\&N) Yellow Ochre Artists' Oil Colour tube paint was used to make model paint films for this study. The binding medium of the paint was safflower oil (stated on the tube), and analysis (see supplementary information for details) confirmed the presence of natural yellow ochre pigment with associated clay minerals (kaolinite and quartz), and hydromagnesite present as an extender.

A custom-built film caster and blade (Sheen instruments) was used to produce homogenous paint films with a wet-film thickness of $300 \mu \mathrm{m}$, and a dry-film thickness of $\sim 260 \mu \mathrm{m}$. The paint films were casted onto Teflon-coated steel plates. The use of Teflon-coated steel as a support enabled paint films of sufficient size, to be detached in order to produce either free-paint films for mechanical testing, or differently mounted samples as required. Immediately after casting, paint films were left to dry for a period of one month while exposed to ambient light and temperature conditions (conditions were not recorded). This was followed by storage in the dark for 9 months (for cross section samples and samples evaluated using DMA and nanoindentation) and 19 months (for samples evaluated using SEM imaging) prior to solvent exposure and analysis. The paint samples were not artificially soiled prior to solvent exposure.

For the Nanoindentation and DMA measurements were carried out two weeks after solvent exposure, when the yellow ochre paint films were $\sim 11$ months old. Owing to instrument availability, SEM imaging was carried out one week after solvent exposure, when the yellow ochre paint films were $\sim 21$ months old.

It is well established that oil paints containing earth pigments have a tendency towards mechanical weakness [10]. In addition, the yellow ochre paint films used in this study were young and relatively poorly crosslinked. As such these test paint samples are likely to be more responsive to solvents than may be expected with the (predominantly) older paint films encountered in conservation practice. The use of safflower oil (a semi-drying oil) as a binding medium will also contribute toward the tendency of the paint to remain poorly crosslinked. Furthermore, yellow ochre pigments do not readily form metal soaps with oil binding media which reduces the tendency of this paint to form a stabilizing ionomeric 
network [29-31]. The use of a non-porous Teflon coated steel plate is also unlike typical primed canvas supports; in this case oxygen access is restricted and the nonabsorbency of the steel is likely to have produced a more medium rich paint surface-potentially adding to its inherent vulnerability.

\section{Selected solvents}

Three key solvent types explored in this study. Water was chosen to represent polar aqueous systems, Shellsol D40 to represent non-polar aliphatic hydrocarbon systems and D5 to represent all silicone-solvent systems, although D2 is also in use in Conservation. As noted in the introduction, these solvents form the basis of many wet-cleaning systems, however, their effect on the physical properties of oil paints have not been explored. See supplementary information for key properties of solvents used in this study (Additional file 1: Table S2).

\section{Solvent exposure}

Yellow ochre paint films were exposed to the selected solvents either by immersion, representing an extreme duration and mode of exposure, or by sponge-cleaning designed to better represent a more typical conservation scenario. For immersion, small pieces of yellow ochre paint films were removed from their Teflon-coated steel support and immersed in the selected solvent for $24 \mathrm{~h}$. The mass of solvent used for immersion was $100 \times$ that of the paint sample.

For sponge-cleaning a Muji ${ }^{\mathrm{TM}}$ makeup sponge (styrenebutadiene rubber) was dipped into a small volume of the solvent $(\sim 4 \mathrm{~mL})$, for $\sim 1-2 \mathrm{~s}$, and blotted on absorbent tissue, before being lightly passed over the upper surface of the paint sample ten times to simulate cleaning. In order to make this process as reproducible as possible across the samples, sponge-cleaning was carried out by the same conservator.

After solvent exposure via immersion or sponge cleaning, samples were left to dry face-up on a Tefloncoated steel sheet. Any further sample-preparation processes that were carried out prior to testing/analysis are described in the relevant section below. For the purpose of this paper, paint films which were not exposed to solvents (24-h immersion or sponge-cleaning) will be referred to as untreated paint films.

\section{Cross section preparation}

A cross section sample was prepared by embedding a small fragment of the untreated paint film in a polyester resin (Tiranti LTD, 'Clear Casting AM' resin) which was cured using a liquid hardener (Butanox M-50; methyl ethyl ketone peroxide solution in dimethyl phthalate, supplied by Tiranti LTD). So as not to expose the paint cross section to solvent, the sample was dry-ground and polished using Micromesh ${ }^{\mathrm{TM}}$ polishing cloths.

\section{Scanning electron microscopy (SEM)}

In order to identify any topographical alterations caused by solvent exposure, the surface of the yellow ochre paint films was studied using scanning electron microscopy (SEM) following either 24-h immersion or sponge-cleaning. SEM imaging was carried out on uncoated paint films using a FEI Quanta 650 FEG SEM in variable pressure mode at $75 \mathrm{~Pa}$ and using a secondary electron (SE) detector operated at $5 \mathrm{kV}$.

Paint samples for SEM surface-imaging were taken from areas of the paint film which were not used for mechanical test samples. They were 21 months old prior to solvent exposure. To ensure that the paint samples were sufficiently dried following solvent exposure and prior to their introduction into the scanning electron microscope, after solvent exposure, samples were mounted onto a carbon adhesive pad fixed to an aluminium stub and heated at $30{ }^{\circ} \mathrm{C}$ for $46 \mathrm{~h}$. Untreated paint films, as well as those which only underwent the heating step, were imaged alongside those exposed to solvents for comparison purposes. SEM surface-imaging took place one week after solvent exposure.

\section{Dynamic Mechanical Analysis (DMA)}

Measurements of bulk mechanical properties of untreated and solvent-treated yellow ochre paints were carried out using a Tritec 2000 Dynamic Mechanical Thermal Analyser (DMA, Triton Technology). Paint samples for DMA analysis were 11 months old at the point of solvent exposure, and measurements were carried out $\sim$ two weeks after solvent exposure. Paint samples prepared for DMA had dimensions of $\sim 1 \mathrm{~cm} \times 3 \mathrm{~cm} \times 240 \mu \mathrm{m}$ with a mass of $\sim 0.2 \mathrm{~g}$. The paint films were tested in tensile mode at a frequency of $1 \mathrm{~Hz}$ and a strain amplitude of $0.02 \%$. The glass transition temperature $\left(T_{\mathrm{g}}\right)$ and moduli were determined by employing a temperature sweep from -10 to $80{ }^{\circ} \mathrm{C}$ at a rate of $2{ }^{\circ} \mathrm{C}$ per minute. At least two replicates for each sample type were examined.

\section{Nanoindentation}

Yellow ochre paint films were exposed to the selected solvents and mounted onto glass slides for the measurement of surface mechanical properties using nanoindentation. Paint samples for nanoindentation were $\sim 11$ months old at the point of solvent exposure, and measurements were carried out $\sim 6$ weeks after exposure. These samples had dimensions of $\sim 1 \mathrm{~cm} \times 1 \mathrm{~cm}$ and a mass of $\sim 0.05 \mathrm{~g}$.

After sponge cleaning, the intrinsic tackiness of the underside of the paint film (which had been in contact 
with the Teflon-coated steel substrate) remained, and a small amount of pressure was used to "self-adhere" the sample to a glass slide. However, samples that had been immersed for 24-h and then allowed to dry on the Teflon-coated steel sheets would no longer self-adhere to the glass slide. To assist with mounting of the immersed samples, a droplet of the same solvent used for the immersion was applied between the glass slide and the sample. However, this mounting process was not effective in all cases. Consequently, no valid data could be obtained for the samples immersed for $24 \mathrm{~h}$ in Shellsol D40 or D5 silicone solvent.

Following the method described in Freeman et al. 2019 [32], dynamic nanoindentation measurements were conducted at ambient conditions $\left(\sim 22{ }^{\circ} \mathrm{C}\right.$ and $\left.50 \% \pm 5 \% \mathrm{RH}\right)$ using an Ultra Nano Hardness Tester (UNHT; Anton Paar) fitted with a three-sided diamond Berkovich indenter. The sample was loaded exponentially with time at a rate of $\sim 0.1 \mathrm{~s}^{-1}$ to maintain constant loading strain rate. After reaching a maximum value, the load on the sample was held constant for $90 \mathrm{~s}$ before being removed linearly at a rate of $1000 \mathrm{mN} / \mathrm{min}$. A sinusoidal load variation with a frequency of $20 \mathrm{~Hz}$ was superimposed on the exponential load function to measure the contact stiffness continuously throughout the loading period. The depth of the pyramidal indenter in contact with the sample surface, or the contact depth $\left(h_{\mathrm{c}}\right)$, was determined as a function of the penetration depth $(h)$, applied load $(P)$ and storage stiffness $(S)$ using the equation proposed by Oliver and Pharr $\left(h_{\mathrm{c}}=h-0.75 P / S\right)$ [33]. The contact depth was converted to an equivalent contact radius $(a)$, using an area function obtained with a reference material of known Young's modulus and Poisson's ratio (fused silica). The reduced storage modulus was calculated as $E_{\mathrm{r}}^{\prime}=S /(2 \beta a)$, where $\beta(=1.034)$ is the geometric constant of the Berkovich indenter.

\section{Results and discussion}

\section{Summary of key observations}

A summary of key findings that will be discussed below are presented in Table 1. A delay in instrument availability meant that samples examined using SEM were 21 months old prior to solvent exposure, whilst samples analysed by cross section, DMA and nanoindentation were 11 months old prior to solvent exposure.

\section{Surface appearance of paints before and after solvent exposure}

The surfaces of untreated yellow ochre paint films were medium rich with a medium-skin visible at the surface. In order to determine the thickness of this mediumrich surface skin, cross-sections were examined (Fig. 1). A UV-fluorescent medium skin of ca. 9-15 microns thickness was visible in the cross section sample taken from the untreated paint. Medium skins are frequently observed in modern oil paints, and are often, but not always, associated with water-sensitivity [34]. It is known that W\&N Yellow Ochre artists' oil paints are prone to becoming sensitive toward water, and that this is promoted under conditions of elevated lux and high relative humidity [14]. For this study, the yellow ochre paint films were classed as non-water-sensitive based on swab-rolling tests performed with ready-made swabs and deionized water, where surface disruption or pigment pickup were not observed [5]. However, there may be a tendency for the curing pathways in the the yellow ochre paint films used in this study to be dominated by peroxyl radicals, given the presence of: $\mathrm{Fe}(\mathrm{III})$ ions present in the paint; the safflower oil binding medium that contains a low proportion of linolenic acid; a lack of exposure to UV light [2]. This may have influenced the response of the paint film toward water, that is described below.

Examination of sponge-cleaned and untreated paint films, using light microscopy and SEM imaging (see Additional file 1: Fig. S5), showed that sponge-cleaning using the three selected solvents did not have any noticeable effect on the visible or surface-topographical characteristics of the paint films.

However, as to be expected, surface changes were observed following the more extreme exposures. Relative to untreated control (Fig. 2a) the images of the surface of the samples subjected to 24-h immersion in deionised water and Shellsol D40 (Figs. 2b, c respectively), revealed a rougher surface topography with some visible 'cratering. Since organic material appears dark in SEM, the lighter and whiter appearance of Figs. $2 b$ and $c$ with respect to Fig. 2a, in conjunction with the observed surface roughening, may suggest a degree of leaching and/ or disruption of organic material at the paint surface. SEM imaging did not show evidence of surface changes following 24-h immersion in D5 silicone solvent relative to the control (Fig. 2d vs. a) with both samples appearing similarly dark (i.e. medium-rich), and smooth. The SEM images were consistent with visual observations of the paint surfaces, where the sample immersed for 24-h in water had developed a distinctly matt and leached appearance relative to the samples immersed in Shellsol D40 which appeared only slightly more matte in comparison to the untreated surface, and the D5 sample which appeared unchanged relative to the control.

The trends described above also tend to align with the DMA and nanoindentation findings (discussed below), i.e. that immersion in deionized water had the most 
Table 1 Summary of key findings for paint aged 11 months (cross section, DMA and Nanoindentation) and 21 months (SEM imaging)

\begin{tabular}{|c|c|c|c|}
\hline \multirow[t]{2}{*}{ Investigation technique } & \multirow[t]{2}{*}{ Sample type } & \multicolumn{2}{|l|}{ Age of paint film (months) } \\
\hline & & 11 & 21 \\
\hline Cross section & Untreated sample & $\begin{array}{l}\text { Untreated sample had a UV-fluorescent medium } \\
\text { skin present at the paint surface; ca. 9-15 } \mathrm{mm} \\
\text { thick }\end{array}$ & $\mathrm{N} / \mathrm{A}$ \\
\hline \multirow[t]{2}{*}{ SEM } & 24-h immersion & N/A & $\begin{array}{l}\text { Surface disruption caused by deinoised water and } \\
\text { shellsol D40. No visible changes caused by D5 }\end{array}$ \\
\hline & Sponge cleaning & $\mathrm{N} / \mathrm{A}$ & $\begin{array}{l}\text { No visible changes to the surface for any of the } \\
\text { selected solvents }\end{array}$ \\
\hline \multirow[t]{2}{*}{$\begin{array}{l}\text { DMA } \\
\text { (bulk mechanical propertie) }\end{array}$} & 24-h immersion & $\begin{array}{l}\text { Deionised water caused a fivefold increase in } \\
\text { storage modulus }\left(E^{\prime}\right) \text { or stiffness; Shellsol D40 } \\
\text { caused a twofold increase in stiffness; there } \\
\text { were no measurable changes to paint stiffness } \\
\text { caused by D5 } \\
\text { Also, differences in } T_{g} \text { were observed based on the } \\
\text { tan } \delta \text { measurements }\end{array}$ & $\mathrm{N} / \mathrm{A}$ \\
\hline & Sponge cleaning & $\begin{array}{l}\text { No significant change of bulk mechanical } \\
\text { properties } E^{\prime} \text { or } T_{\mathrm{g}} \text { was measured for any of the } \\
\text { selected solvents }\end{array}$ & N/A \\
\hline \multirow[t]{3}{*}{$\begin{array}{l}\text { Nanoindentation (surface } \\
\text { mechanical properties) }\end{array}$} & Untreated sample & $\begin{array}{l}\text { There is considerable variation in measured } \\
\text { reduced storage modulus }\left(E_{r}^{\prime}\right) \text { and loss } \\
\text { tangent, (tan } \delta \text { ) across the surface at a given } \\
\text { measurement depth, owing to the inherent } \\
\text { variability of the surface } \\
\text { The reduced storage modulus, }\left(E_{r}^{\prime}\right) \text { and loss tan- } \\
\text { gent (tan } \delta \text { ) increase and decrease respectively } \\
\text { with increasing depth. This is consistent with } \\
\text { the surface having a lower PVC, and being less } \\
\text { stiff and more medium rich compared to bulk } \\
\text { paint }\end{array}$ & $\mathrm{N} / \mathrm{A}$ \\
\hline & 24-h immersion & $\begin{array}{l}\text { Reduced storage modulus }\left(E_{r}^{\prime}\right) \text { or stiffness } \\
\text { increased fivefold following immersion in } \\
\text { water }\end{array}$ & $\mathrm{N} / \mathrm{A}$ \\
\hline & Sponge cleaning & $\begin{array}{l}\text { No significant changes to surface mechanical } \\
\text { properties i.e. reduced storage modulus }\left(E_{r}^{\prime}\right) \text { or } \\
\text { loss tangent (tan } \delta \text { ) were detected follow- } \\
\text { ing sponge cleaning with either of the three } \\
\text { selected solvents }\end{array}$ & N/A \\
\hline
\end{tabular}
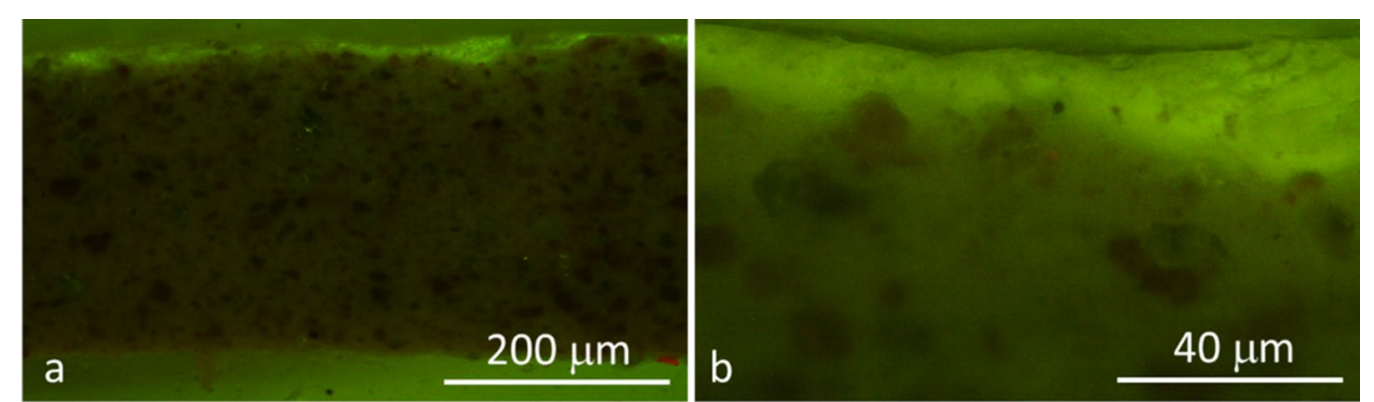

Fig. 1 Images of a cross-section of untreated, 10 months old yellow ochre oil paint, under UV-light illumination

pronounced effect on the paint films, and that sponge cleaning had no discernible effect on the samples (see SEM images in Additional file 1: Fig. S5), regardless of which of the selected solvents were used. 

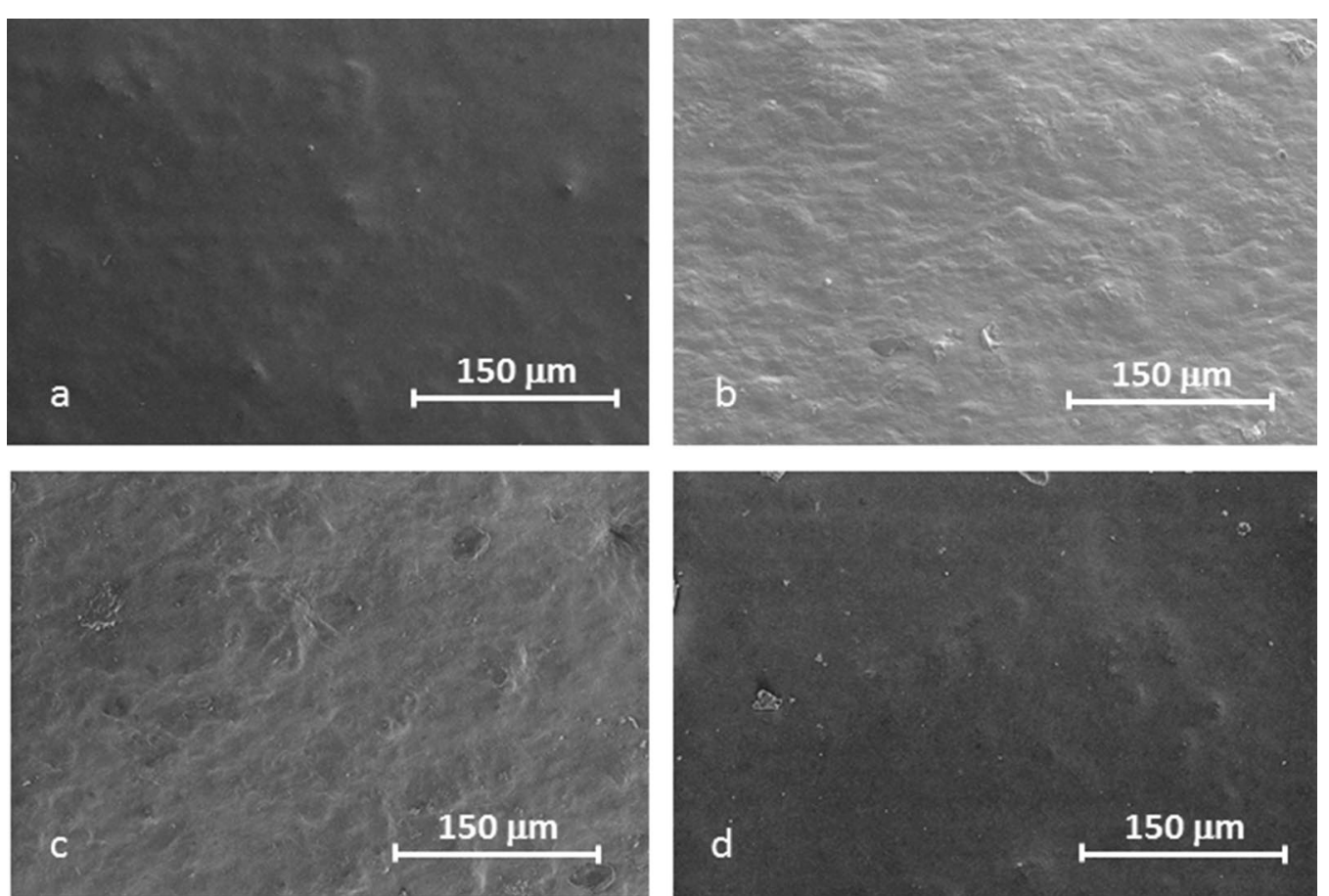

Fig. 2 Secondary electron SEM images at $150 \times$ magnification, of 21 month old Yellow Ochre oil paint films, after heating at $30^{\circ} \mathrm{C}$ for $46 \mathrm{~h}$ following a untreated sample, b 24-h immersion in deionised water, c 24-h immersion in Shellsol D40, d 24-h immersion in D5 silicone solvent

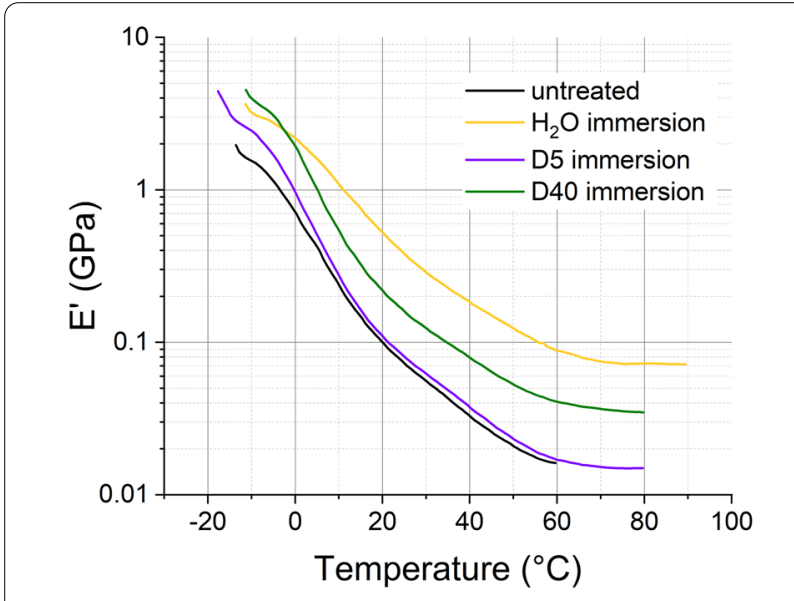

Fig. 3 Storage modulus for free film samples immersed in different solvents. Each curve represents average of two temperature scans

\section{Measurement of bulk-mechanical properties using Dynamic Mechanical Analysis}

The bulk mechanical properties of untreated and treated (sponged and immersed) paint films were examined using Dynamic Mechanical Analysis. DMA tests were performed to quantify storage modulus ( $E^{\prime}$, stiffness) and loss tangent $(\tan \delta)$ over a range of temperatures $(-10$ to $80{ }^{\circ} \mathrm{C}$ ) (Figs. 3 and 4 ). Figure 3 presents the storage

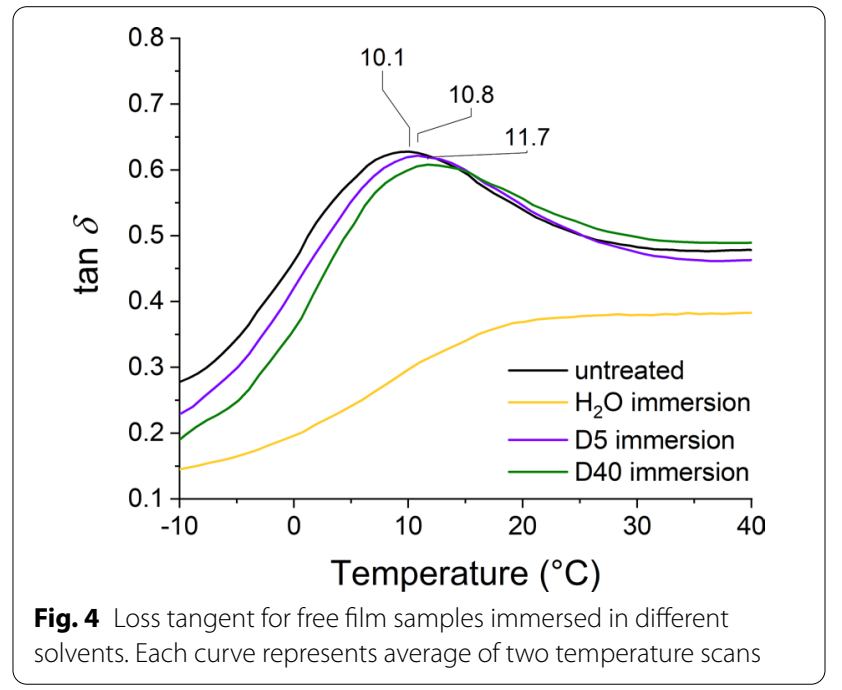

modulus $E^{\prime}$ versus temperature for the untreated and immersed samples. At room temperature $\left(\sim 20^{\circ} \mathrm{C}\right)$, DMA tests revealed an increase in stiffness of paint samples due to immersion in deionised water ( fivefold) and Shellsol D40 immersion ( twofold), and minimal change due to immersion in D5 silicone solvent. The increase in stiffness, which is signified by a higher storage modulus, is most likely the result of leaching of non-crosslinked 
constituents present in the binding medium of this paint film. These observations are consistent with the general trends shown in the secondary electron SEM images of the untreated and immersed samples (Fig. 2). The greatest change in $E^{\prime}$ was observed for the deionised water immersed sample which was accompanied by a substantial decrease in $\tan \delta$ (or viscosity) from $\sim 0.55$ to $\sim 0.37$, at room temperature $\left(20{ }^{\circ} \mathrm{C}\right)$, as shown in Fig. 4 . Immersion in Shellsol D40 or D5 silicone solvent was also found to affect the $\tan \delta$ of the bulk film material but to a much lesser degree. The glass transition temperatures or $T_{\mathrm{g}}$ of the yellow ochre sample before and after immersion are shown in Fig. 4. The untreated sample has the lowest $T_{\mathrm{g}}$ $\left(10.1^{\circ} \mathrm{C}\right)$ and the largest $\tan \delta$ peak. Additional leaching of lipidic components is known to shift the $T_{\mathrm{g}}$ to higher temperatures. After immersion in D5 silicone solvent and Shellsol D40 the $T_{\mathrm{g}}$ shifted slightly to $10.8^{\circ} \mathrm{C}$ and $11.7^{\circ} \mathrm{C}$, respectively. Whereas after immersion in deionized water the maxima of the $\tan \delta$ peak decreased considerably, making it more difficult to determine the numerical value of $\mathrm{Tg}$. However, an increase in Tg toward a higher temperature was observed.

In contrast to the immersed samples, the $E^{\prime}$ and $\tan \delta$ of the sponge-cleaned samples showed no significant change compared to that of the untreated sample (Additional file 1: Figs. S6 and S7). This directly indicates that sponge-cleaning of the paint surface, with the selected solvents, had an insignificant effect on the bulk mechanical properties of the paint film. This was also consistent with visual and SEM evaluation of the paint surfaces after sponge-cleaning as described above (see Additional file 1: Fig. S5).

\section{Mechanical properties of the subsurface using nanoindentation}

Nanoindentation testing was performed for the untreated, sponge-cleaned (with all three solvents), and deionized water immersed samples. In addition, drysponge cleaning was also evaluated in order to investigate the effect of mechanical action on the paint surface. Nanoindentation of all tested samples, performed at either $10,12,13$ or 16 locations across the surface of the sample, revealed a considerable scatter of reduced storage modulus $\left(E_{\mathrm{r}}{ }^{\prime}\right)$ and loss tangent $(\tan \delta)$ at given indentation depths. The observed scatter is attributed to the surface roughness, material heterogeneity including variations in the thickness of the medium skin, and the non-uniform distribution of small and hard pigments throughout a soft binding medium. The general inhomogeneity of the surface, which becomes all the more essential on the micro scale, is visible using SEM imaging (Fig. 5).

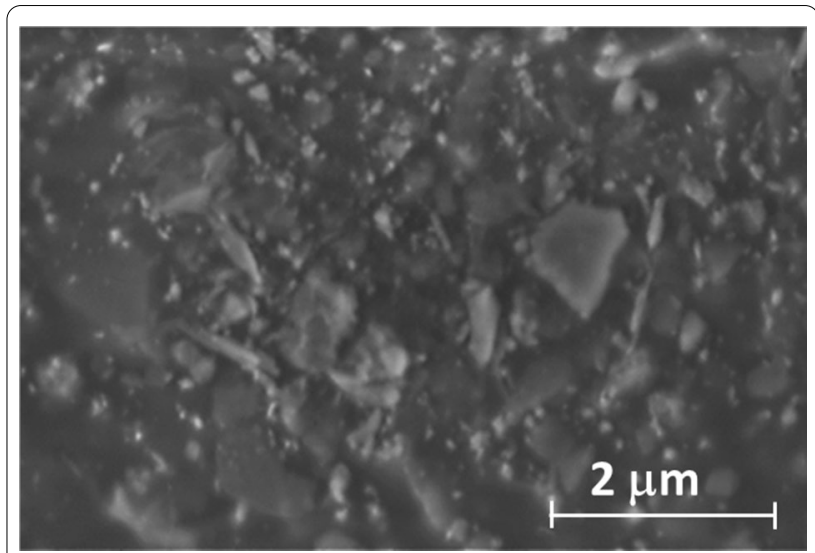

Fig. 5 Secondary electron SEM image at 10,000 $\times$ magnification of a 21-month-old, untreated yellow ochre paint film, showing the distribution of pigment and extender particles / aggregates within the binding medium

As a result of the local roughness and inhomogeneity of the surface, some of the indentation measurements were deemed to have suffered from considerable over- or under-estimation of the effective contact point of the indenter tip with the sample surface. The storage stiffness versus depth curve of each indentation test was thus inspected, and obvious outliers as well as those exhibiting kinks were excluded from analysis (see Additional file 1: Fig. S8). This selection process reduced the number of valid tests $(n)$ to 7 for all samples except for the dry-sponge-cleaned sample that had 4 valid tests. The indentation results of each sample were analysed at the penetration depths of 1,2 and $3 \mu \mathrm{m}$. Since the nanoindentation measurement at a given penetration depth reflects the mechanical properties of the local volume several times deeper into the material, the effective sampling volume at testing depths of $3 \mu \mathrm{m}$ also encompasses the properties of the material several times deeper into the material (e.g. 15-20 $\mu \mathrm{m})$. At these depths, this embodies the most medium rich surface of the paint, which is typically most vulnerable during cleaning.

The $E_{\mathrm{r}}^{\prime}$ and $\tan \delta$ of the untreated sample $(n=7)$ increased and decreased, respectively, with increasing penetration depth, as shown in Fig. 6. This together with the near-surface $\tan \delta$ values close to unity implies that the local PVC of the untreated material is lowest in the subsurface, being dominated by the oil-rich binding medium and increases progressively with depth as with the local material heterogeneity. This is consistent with the observation of a surface medium skin (see Fig. 1) which appears to vary in thickness of ca. 9-15 microns in depth.

Figure 7 shows the $E_{\mathrm{r}}{ }^{\prime}$ versus $\tan \delta$ of the sample after immersion in deionized water $(n=7)$, which is 


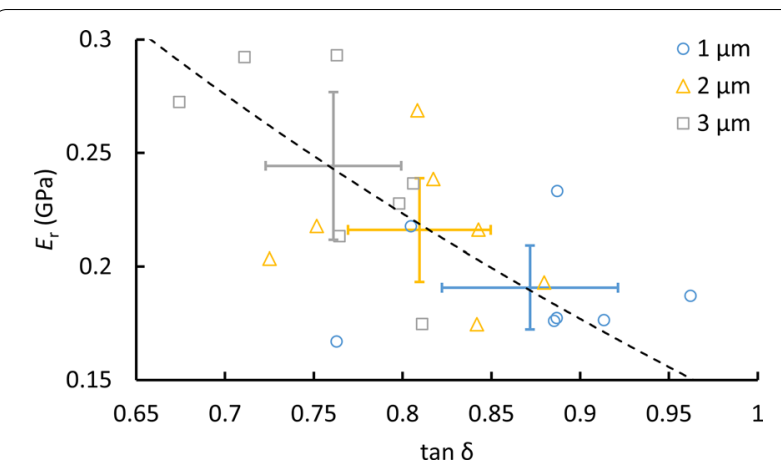

Fig. 6 Reduced storage modulus versus loss tangent of control sample at the depths of 1,2 and $3 \mu \mathrm{m}$, where each marker shows the results of an individual test at either depth with correspondingly coloured error bars indicating the $95 \%$ confidence intervals at that depth (assuming a normal distribution). The dashed line is a logarithmic function fitted to the averaged results at the three depths

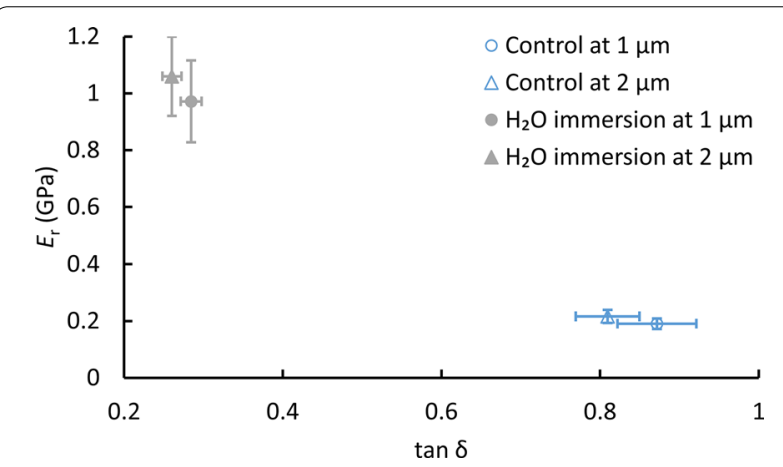

Fig. 7 Reduced storage modulus versus loss tangent of control and water-immersed samples at 1 and $2 \mu \mathrm{m}$, with each marker and associated error bars showing the mean values and $95 \%$ confidence intervals, respectively

statistically significantly different from those of the untreated sample, at the depth of 1 and $2 \mu \mathrm{m}$. After immersion the sample became noticeably stiffer, and $\mathrm{a} \sim$ fivefold increase in modulus as a result of deionised water immersion is in agreement with the DMA (yellow curve in Fig. 3). Unlike the untreated sample, data was not obtained at the depth of $3 \mu \mathrm{m}$ as the indenter was unable to penetrate deep enough into the deionised water-immersed sample at the maximum applied load of $5 \mathrm{mN}$.

The magnitude of the modulus of the deionized-waterimmersed sample as measured by nanoindentation was approximately twice that measured by DMA. This may be attributed to 20 times greater excitation frequency used in nanoindentation testing in comparison to DMA. Also, nanoindentation testing is largely compressive in contrast to the solely tensile DMA testing. The $\sim$ fivefold increase in modulus, shown in Fig. 7, as a result of deionised

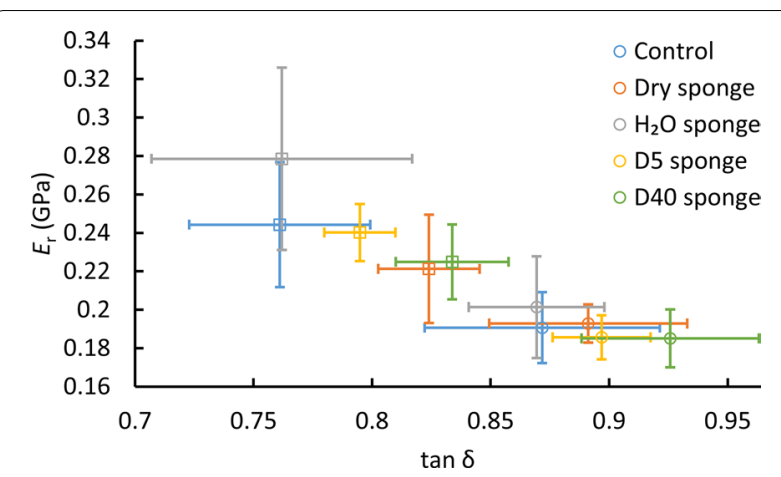

Fig. 8 Reduced storage modulus versus loss tangent of control and sponge-cleaned samples at the depths of $1 \mu \mathrm{m}$ (circles) and $3 \mu \mathrm{m}$ (squares), with each marker and associated error bars showing the mean values and $95 \%$ confidence intervals, respectively

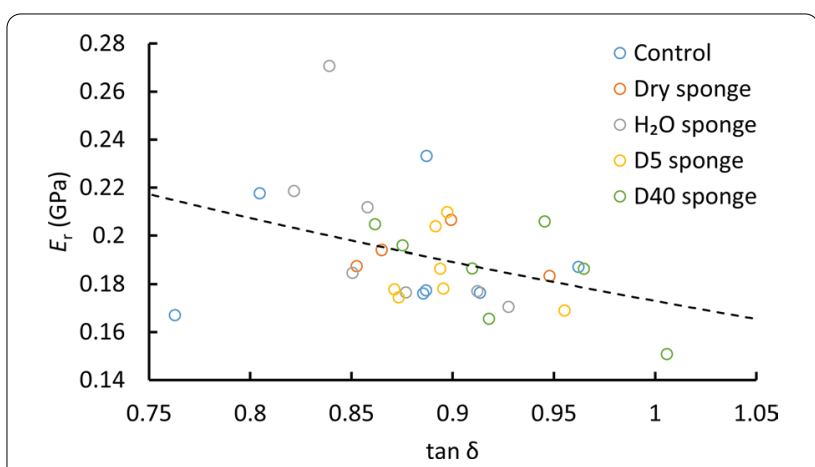

Fig. 9 Reduced storage modulus versus loss tangent of control and sponge-cleaned samples at $1 \mu \mathrm{m}$, where each marker shows the results of an individual test of a sample, and the dashed line is a logarithmic function fitted to all results of all five samples $(n=32)$

water-immersion reflects the increased stiffness of the paint, as previously discussed.

The $E_{\mathrm{r}}^{\prime}$ versus $\tan \delta$ of the untreated and spongecleaned samples at the depths of 1 and $3 \mu \mathrm{m}$ are plotted in Fig. 8. The overlapping or nearly overlapping error bars of the untreated sample with those of the sponge-cleaned samples at either depth suggest that there is no significant change in the viscoelastic properties of the oil paint material as a result of sponge cleaning. Due to the small sample size of $n=7$ (or $n=4$ for the dry-sponge-cleaned sample), however, it is difficult to confirm that the effect of sponge cleaning is statistically non-significant. To better assess the effect of each type of sponge cleaning on the surface mechanical properties of the paint material, the following analysis was performed.

The $E_{\mathrm{r}}{ }^{\prime}$ and $\tan \delta$ values of all valid tests at the depth of $1 \mu \mathrm{m}$ are plotted in Fig. 9. The $E_{\mathrm{r}}{ }^{\prime}$ and $\tan \delta$ results at the shallowest depth are plotted because this is where a cleaning effect should be most pronounced, and this is where subtle changes in mechanical properties are most 
readily detectable by nanoindentation. The dashed line in the figure is a logarithmic function fitted to all valid data of all five samples at the same depth of $1 \mu \mathrm{m}(n=32)$, showing an inverse relationship between the modulus and loss tangent. This function is intended to show a trend in the obtained data, and does not represent any physical model describing the tested materials. The fact that the data from the untreated and each of the spongecleaned samples (excluding the two outliers much further apart from the trend line) are evenly spread around the same inverse relationship across similar data ranges indicates that the mechanical effect of a sponge-cleaning procedure was smaller than the inherent variability in the mechanical properties of the original paint material itself, comprised of hard pigments distributed in a random manner in a soft binding medium.

\section{Conclusions}

The mechanical properties of naturally aged Winsor \& Newton yellow ochre oil paint films were measured following 24-h immersion and sponge cleaning using deionized water, Shellsol D40 and silicone solvent D5. Dynamic Mechanical Analysis was used to measure bulk mechanical properties in tensile mode. Nanoindentation enabled the evaluation of the effects of solvent exposure on the surface mechanical properties of the uppermost microns of the paint surface.

Both DMA and nanoindentation indicated $a \sim$ fivefold increase in paint stiffness following 24-h immersion in deionised water, i.e. a similar increase in paint stiffness occurred at both the bulk and the surface. This represented the largest change in mechanical properties observed in this study; and concurs with empirical observations i.e. that the paints immersed in water became noticeably more brittle with a tendency to crack upon handling. DMA analysis suggested a twofold increase in bulk paint stiffness following 24-h immersion in Shellsol D40 and negligible change in paint stiffness following 24-immersion in silicone solvent D5. Additionally, the slight shift in the height of the tan delta peak after immersion in D40 and D5 can be attributed to extraction of mobile material, however, further exploration is needed. Furthermore, DMA demonstrated a strong mechanical dependence on temperature, hence it would also be useful to carry out similar experiments with varied ambient temperature and relative humidity.

The immersion experiments indicate that the young yellow ochre paint film was most vulnerable to changes in mechanical properties caused by prolonged exposure to water. This is likely to relate to the hydrolysis of ester bonds which would influence the mechanical properties of the paint film. The vulnerability toward water may also partly relate to the hygroscopic clay mineral content of yellow ochre, which is known to promote the adsorption and transport of water into the bulk paint [35]. Although the paint films at the time of this study were not classed as water sensitive based on aqueous swab rolling, yellow ochre W\&N oil paint is amongst those known to have a tendency to develop water sensitivity over time [7]. Therefore the question arises as to whether the initial curing behaviour [2] of this paint film could also contribute towards the reponse to water. In the case of oil paint, water is typically not considered a strongly swelling solvent, nor one into which water readily diffuses[15]. However future studies that investigate the diffusion, swelling and transport mechanisms of water for modern oil paints associated with the development of water sensitivity would be of merit.

The fact that Shellsol D40 had a greater effect on bulk mechanical properties than silicone solvent D5 may be attributed in part to Shellsol D40 having a higher vapour pressure as well as likely differences in relative fatty acid solubility in each solvent. Higher vapour pressure solvents are known to cause a higher degree of leaching of free fatty acids, diacids and glycerides [35]. Moreover, Shellsol D40 is more likely to extract different amounts and proportions of the mobile lipidic phase within the paint film relative to D5. Further research into the transport mechanisms and interactions of these solvents within oil paint films would be required to better understand the changes in mechanical properties identified in this study. Furthermore the potential influence of solvent-retention on measured mechanical properties requires further investigation; Ormsby et al. [14] identified the presence of D5 in a yellow ochre oil paint film two weeks after cleaning, consequently, D5 and D40 may still be present at low levels within the films examined in this study.

When the selected solvents were applied by sponge (ten strokes) or where dry sponges were used, nanoindentation and DMA measurements detected no significant changes to the bulk or surface mechanical properties. Similarly, no surface changes were detectable using secondary electron SEM imaging. The use of these three evaluation techniques has confirmed the empirical observations noted by practitioners that shorter solvent exposure durations, and the use of sponges, are less likely to result in changes to the paint bulk and surface mechanical properties despite the inherent difference in sensibility demonstrated by the immersion tests. In the previous study by Hedley et al. [13] the effect of more aggressive solvents and solvent application on 12-yearold lead white and burnt sienna linseed oil films were examined. Both 24-h immersion, in water, propanol, or acetone, and swabbing cleaning, for 1,3 , or $5 \mathrm{~min}$, showed differences in thermomechanical properties and leaching 
of polar components. Whereas Mecklenburg, Tumosa, and Erhardt [19] examined the effect of short-term exposure $(60 \mathrm{~s})$ in acetone of a 7.5-year-old titanium dioxide linseed oil film. They found that $60 \mathrm{~s}$ of immersion in acetone was long enough to leach mobile materials resulting in an increase in stiffness and strength. Within this study, the modern solvent choices, and the gentler application method of sponge cleaning, resulted in a limited mechanical effect on the surface, stressing the importance of focusing on appropriate solvents and application systems.

This pilot study demonstrates a combined analytical approach that can be utilized for developing our understanding of the effect on mechanical properties of exposure to cleaning materials on paint films. The short-term exposure to solvents mimics conservation treatment more closely than relying solely on immersion studies as per past research. This study was limited to evaluation of the effect of three key solvents, and one pigment type and brand of oil paint. In order to provide useful guidance for conservators, a wider range of cleaning materials and paint materials should be investigated in the future. Furthermore, the influence of the rate of diffusion of the selected solvents into the paint film, the inherent solubility of lipidic materials in these solvents and solvent retention should also be investigated.

\section{Supplementary Information}

The online version contains supplementary material available at https://doi. org/10.1186/s40494-021-00501-8.

Additional file 1: Figure S1. EDX spectrum of Winsor \& Newton artists' oil colour tube paint. Fe and $\mathrm{O}$ suggest an iron oxide e.g. Fe2O3; Al, Si and O suggest aluminosilicate or quartz present as clay minerals; magnesium, and carbon is attributed to the presence of hydromagnesite, identified using FTIR i.e. Mg5(CO3)4(OH)2; trace elements $\mathrm{Na}$, $\mathrm{K}$, $\mathrm{Ca}$, Ti are known to occur in natural ochres. See: Genestar C, Pons C. Earth pigments in painting: Characterisation and differentiation by means of FTIR spectroscopy and SEM-EDS microanalysis. Anal Bioanal Chem. 2005. Figure S2. transmission FTIR spectrum of dried and untreated Winsor \& Newton artists' oil colour tube paint. Figure S3. Image of yellow ochre paint films on Teflon-coated steel supports. The paint film on the left has been peeled off the support and placed bottom-face-up. The paint film on the right-hand side is face-up. This illustrates that the surface is medium rich and glossy relative to the underside. Figure S4. Hirox images all at 500x mag, of the surfaces of yellow ochre paint films following 24-hours in the selected solvents. Top left: untreated sample; Top right: 24-hour immersion in deionised water; bottom left: 24-hour immersion in Shellsol D40; bottom right: 24-hour immersion in D5 silicone solvent. The Hirox images show the greatest surface change was following exposure to deionised water. The surface topography is more defined and coarser. An illusion of a 'glossy' surface is produced in the BR image owing to diffusive reflection from the matte, uneven surface. Figure S5. Secondary electron SEM images of Yellow Ochre oil paint films, all at $150 \times$ magnification (a) no solvent-exposure (b) sponge-cleaning using deionised water (c) spongecleaning using Shellsol D40 (d) sponge-cleaning D5 silicone solvent. The horizontal bands visible in images $b$-d are due to interference during imaging and were not caused by the sponge cleaning. Figure S6. Storage modulus for free film samples sponge-cleaned with different solvents. Each curve represents average of two temperature scans. Figure S7. Loss tangent for free film samples sponge-cleaned with different solvents. Each curve represents average of two temperature scans. Figure S8. Storage stiffness versus displacement $(n=12)$ of the control sample measured by nanoindentation, where the five experiments indicated in colour other than grey were excluded from analysis, being identified as outliers or due to kinks, likely resulting from the local roughness or inhomogeneity of the surface. Table S1. Summary of band assignments for the FTIR spectrum of W\&N Yellow Ochre oil paint, shown in Figure S2. The dominant presence of clay minerals and lack of gypsum is consistent with a natural French ochre. See: Genestar C, Pons C. Earth pigments in painting: Characterisation and differentiation by means of FTIR spectroscopy and SEM-EDS microanalysis. Anal Bioanal Chem. 2005. Table S2. Some key properties of the solvents used in this study, where $\mathrm{fd}=$ dispersion forces, $\mathrm{fp}=$ polarity, and $\mathrm{fh}=$ hydrogen bonding.

\section{Abbreviations}

DMA: Dynamic Mechanical Analysis; DVS: Dynamic Vapour Sorption; W\&N: Winsor \& Newton; fd: dispersion forces; fp: polarity; fh: hydrogen bonding; SEM: Scanning electron microscopy; SE: Secondary electron; $T_{\mathrm{g}}$ : Glass transition temperature; UNHT: Ultra Nano Hardness Tester; $n$ : Number of valid tests; $E_{r}^{\prime}$ : Reduced storage modulus; $\tan \delta$ : loss tangent.

\section{Acknowledgements}

The authors would like to acknowledge and thank the following people and institutions: Dr Alex Ball (Natural History Museum, London), Dr Joyce Townsend (Tate), and Tom Learner (Getty Conservation Institute).

\section{Authors' contributions}

AAF: Conceptualization, Methodology, Validation, Investigation, Writing original draft, Writing — review \& editing. JL: Conceptualization, Methodology, Validation, Investigation, Writing —original draft, Writing — review \& editing. CKA: Conceptualization, Methodology, Project administration, Writing - original draft, Writing — review \& editing. NF: Conceptualization, Methodology, Validation, Formal analysis, Writing —original draft, Writing—review \& editing. Mł: Conceptualization, Methodology, Validation, Investigation, Project administration, Resources, Writing — original draft, Writing — review \& editing. BO: Conceptualization, Methodology, Resources, Writing — original draft, Writing review \& editing. All authors read and approved the final manuscript.

\section{Funding}

The research has been supported by the Getty Conservation Institute's Managing Collections Environments Initiative.

\section{Availability of data and materials}

All data is available within the manuscript and supplementary information.

\section{Competing interests}

The authors declare that they have no competing interests.

\section{Author details}

${ }^{1}$ The Getty Conservation Institute, Los Angeles, USA. ${ }^{2}$ Tate Gallery, London, UK. ${ }^{3}$ The Royal Danish Academy of Fine Arts, Schools of Architecture, Design and Conservation (KADK), Copenhagen, Denmark.

Received: 19 September 2020 Accepted: 12 February 2021

Published online: 04 March 2021

\section{References}

1. Van den Berg KJ, Burnstock A, De Keijzer M, Krueger J, Learner T, De Tagle A, et al. Issues in contemporary oil paint. New York: Springer; 2014.

2. Bonaduce I, Duce C, Lluveras-Tenorio A, Lee J, Ormsby B, Burnstock A, et al. Conservation issues of modern oil paintings: a molecular model on paint curing. Acc Chem Res. 2019;52(12):3397-406.

3. Bay L, Burnstock A, Lee J, Ormsby B, van der Berg KJ. Water sensitivity of modern oil paintings. In: Proceedings of the ICOM-CC 18th Triennial conferences preprints, September 4-8, Copenhagen; 2017. 
4. Cooper A, Burnstock A, van den Berg KJ, Ormsby B, et al. Water sensitive oil paints in the twentieth century: a study of the distribution of watersoluble degradation products in modern oil paint films. In: Van Den Berg KJ, Burnstock A, De Keijzer M, Kreuger J, Learner T, De Tagle A, et al., editors. Issues in contemporary oil paint. Cham: Springer; 2014.

5. Mills $L$, Burnstock A, de Groot S, Megens L, Bisschoff M, van Keulen H, et al. Water sensitivity of modern artists' oil paints. In: Proceedings of the ICOM-CC 15th Triennial conferences preprints, September 22-26, New Delhi; 2008. II. p. 651-659

6. Chung JY, Ormsby B, Lee J, Burnstock A, Van den Berg KJ. An Investigation options for surface cleaning unvarnished water-sensitive oil paints based on recent developments for acrylic paints. In: Proceedings of the ICOMCC 18th Triennial conferences preprints, September 4-8, Copenhagen; 2017.

7. Lee J, Bonaduce I, Modugno F, La Nasa J, Ormsby B, Van Den Berg KJ. Scientific investigation into the water sensitivity of twentieth century oil paints. Microchem J. 2018;138:282-95.

8. Hintz M, van den Berg KJ, Stols-Witlox M, Steyn L. Improving the surface cleaning of water sensitive oil paint by use of alternative application methods. In: van den Berg KJ, Bonaduce I, Burnstock A, Ormsby B, Scharff M, Carlyle L, et al., editors. Conservation of modern oil paintings. Cham: Springer International Publishing; 2019. p. 575-86.

9. Bierings J, Steyn L, Stols-Witlox M, van den Berg KJ. Challenges of surface cleaning paintings by Asger Jorn (1914-1973): an inventory of existing practice. In: van den Berg KJ, Bonaduce I, Burnstock A, Ormsby B, Scharff $M$, Carlyle L, et al., editors. Conservation of modern oil paintings. Cham: Springer International Publishing; 2019. p. 363-72.

10. Tumosa CS, Mecklenburg MF. Oil paints: the chemistry of drying oils and the potential for solvent disruption. new insights into the cleaning of paintings. In: Proceedings from the cleaning 2010 international conference, Universidad Politecnica de Valencia and Museum Conservation Institute Smithsonian Institution; 2013. p. 52-58.

11. Hagan EWS, Murray A. Effects of water exposure on the mechanical properties of early artists' acrylic paints. Materials Issues in Art and Archaeology VII. Boston: Materials Research Society; 2005. p. 1-7.

12. Doménech-Carbó MT, Silva MF, Aura-Castro E, Doménech-Carbó A, Fuster-López L, Gimeno-Adelantado JV, et al. Multitechnique approach to evaluate cleaning treatments for acrylic and polyvinyl acetate paints. In: Mecklenburg M, Charola A, Koestler R, editors., et al., New Insights into the Cleaning of Paintings: Proceedings from the Cleaning 2010 International Conference. Washington, DC: Universidad Politecnica de Valencia and Museum Conservation Institute; 2013. p. 125-34.

13. Hedley G, Odlyha M, Burnstock A, Tillinghast J, Husband C. A study of the mechanical and surface properties of oil paint films treated with organic solvents and water. Stud Conserv. 1990;35(sup 1):98-105.

14. Ormsby B, Lee J, Bonaduce I, Lluveras-Tenorio A. Evaluating cleaning systems for use on water sensitive modern oil paints: a comparative study. In: van den Berg KJ, Bonaduce I, Burnstock A, Ormsby B, Scharff M, Carlyle $L$, et al., editors. Conservation of modern oil paintings. Cham: Springer International Publishing; 2019. p. 11-35.

15. Baij L, Hermans J, Ormsby B, Noble P, ledema P, Keune K. A review of solvent action on oil paint. Heritage Science. 2020;8(1):43.

16. Sutherland K. Solvent leaching effects on aged oil paints. New insights into the cleaning of paintings. In: Proceedings from the cleaning 2010 international conference, Universidad Politecnica de Valencia and Museum Conservation Institute; 2013. p. 45-49.

17. Phenix A. The swelling of artists' paints by organic solvents and the cleaning of paintings: recent perspectives, future directions. In: Mar Parkin $\mathrm{H}$, editor. Postprints of annual conference of the american institute for conservation, Paintings Specialty Group, Miami, Florida, June 6-11, 2002. Washington, D.C.: American Institute for Conservation; 2003. p. 71-86.

18. van den Berg JDJ, van den Berg KJ, Boon JJ. Identification of non-crosslinked compounds in methanolic extracts of cured and aged linseed oil-based paint films using gas chromatography-mass spectrometry. J Chromatogr A. 2002;950(1):195-211.

19. Mecklenburg MF, Tumosa CS, Erhardt D. The changing mechanical properties of aging oil paints. In: Murray L, Vandiver PB, Mass $J \mathrm{~L}$, editors.
Materials issues in art and archaeology VII, Materials Research Society Symposium Proceedings. Pittsburgh: Materials Research Society; 2005. p. 13-24.

20. Gillman M, Lee J, Ormsby B, Burnstock A. Water-sensitivity in modern oil paintings: trends in phenomena and treatment options. In: van den Berg KJ, Bonaduce I, Burnstock A, Ormsby B, Scharff M, Carlyle L, et al., editors. Conservation of modern oil paintings. Cham: Springer International Publishing; 2019. p. 477-94.

21. Bartoletti A, Barker R, Chelazzi D, Bonelli N, Baglioni P, Lee J, et al. Correction to: Reviving WHAAM! a comparative evaluation of cleaning systems for the conservation treatment of Roy Lichtenstein's iconic painting. Heritage Sci. 2020;8:9.

22. Andersen CK, Freeman A, Mortensen MN, Beltran V, Łukomski M, Phenix A. Mechanical and moisture sorption properties of commercial artists' oil paint by dynamic mechanical thermal analysis (DMA), nanoindentation, and dynamic vapour sorption (DVS). In: van den Berg KJ, Bonaduce I, Burnstock A, Ormsby B, Scharff M, Carlyle L, et al., editors. Conservation of modern oil paintings. Cham: Springer International Publishing; 2019. p. 403-418.

23. Dillon CE, Lagalante AF, Wolbers RC. Acrylic emulsion paint films: The effect of solution $\mathrm{pH}$, conductivity, and ionic strength on film swelling and surfactant removal. Stud Conserv. 2014;59(1):52-62.

24. Ormsby B, Kampasakali E, Learner T. Surfactants and acrylic dispersion paints: evaluating changes induced by wet surface cleaning treatments. New insights into the cleaning of paintings Proceedings from the Cleaning 2010 International Conference Universidad Politécnica de Valencia and Museum Conservation Institute. 2013:159-64.

25. Ormsby B, Keefe M, Phenix A, Von Aderkas E, Learner T, Tucker C, et al. Mineral spirits-based microemulsions: A novel cleaning system for painted surfaces. J Am Inst Conserv. 2016;55(1):12-31.

26. Stavroudis C. More from CAPS3: Surfactants, silicone-based solvents, and microemulsions. WAAC Newsl. 2012;34(3):24-7.

27. Myers JM, Cushman M. Analysis of cleaning efficacy and clearance of silicone-based pickering-type emulsions used in the cleaning of watermiscible oil paints. In: van den Berg KJ, Bonaduce I, Burnstock A, Ormsby B, Scharff M, Carlyle L, et al., editors. Conservation of modern oil paintings. Cham: Springer International Publishing; 2019. p. 565-574.

28. Lagalante A, Wolbers R. Particle-based silicone cleaning emulsions: studies in model paint systems for the cleaning of water-sensitive artworks. In: Angelova LV, Ormsby B, Townsend J, Wolbers R, editors. Gels in the conservation of art. London: Archetype Publications Ltd.; 2017. p. 193-9.

29. Hermans J, Keune K, van Loon A, Corkery R, ledema P. Ionomer-like structure in mature oil paint binding media. RSC Adv. 2016;6:93363-9.

30. Mazzeo R, Prati S, Quaranta M, Joseph E, Kendix E, Galeotti M. Attenuated total reflection micro FTIR characterisation of pigment-binder interaction in reconstructed paint films. Anal Bioanal Chem. 2008;392(1-2):65-76.

31. Van der Weerd J, Van Loon A, Boon JJ. FTIR studies of the effects of pigments on the aging of oil. Stud Conserv. 2005;50(1):3-22.

32. Freeman $\mathrm{A}$, Łukomski M, Beltran V. Mechanical characterization of a cross-sectional TiO2 acrylic-based paint by nano-indentation. J Am Inst Conserv. 2020;59(1):27-39.

33. Oliver WC, Pharr GM. Measurement of hardness and elastic modulus by instrumented indentation: advances in understanding and refinements to methodology. J Mater Res. 2004;19(1):3-20.

34. Burnstock A, Van Den Berg KJ. Twentieth century oil paint the interface between science and conservation and the challenges for modern oil paint research. In: Van Den Berg KJ, Burnstock A, De Keijzer M, Krueger J, Learner T, Tagle DA, et al., editors. Issues in contemporary oil paint. Cham: Springer International Publishing; 2014. p. 1-19.

35. Zumbühl S. Parametrization of the solvent action on modern artists' paint systems. Stud Conserv. 2014;59(1):24-37.

\section{Publisher's Note}

Springer Nature remains neutral with regard to jurisdictional claims in published maps and institutional affiliations. 